\title{
Bed Moves, Ward Environment, Staff Perspectives and Falls for Older People with High Falls Risk in an Acute Hospital: A Mixed Methods Study
}

This article was published in the following Dove Press journal:

Clinical Interventions in Aging

\author{
Christine Toye, (iD) ${ }^{\prime}$ \\ Susan Slatyer, (iD) ${ }^{2}$ Su Kitchen, (iD) ${ }^{3}$ \\ Katharine Ingram, (iD) \\ Mary Bronson, (iD ${ }^{5}$ \\ Deborah Edwards, (iD ${ }^{6}$ \\ Welma van Schalkwyk, ${ }^{7}$ \\ Catherine Pienaar, ${ }^{8}$ \\ Philippa Wharton, 9 \\ Chrianna Bharat, (D) 10 \\ Keith D Hill (D) 1
}

'School of Nursing, Midwifery and Paramedicine, Curtin University, Perth, Western Australia 6102, Australia, Centre for Nursing Research, Sir Charles Gairdner Hospital, Perth, Western Australia 6009 , Australia; ${ }^{2}$ Discipline of Nursing, College of Science, Health, Engineering \& Education, Murdoch University, Perth, Western Australia 6150, Australia, Centre for Nursing Research, Sir Charles Gairdner Hospital, Perth, Western Australia 6009, Australia; ${ }^{3}$ Clinical Lead and Clinical Nurse Consultant in Falls

Management, Sir Charles Gairdner Hospital, Perth, Western Australia 6009, Australia; ${ }^{4}$ Consultant

Geriatrician, Sir Charles Gairdner Hospital, Perth, Western Australia 6009, Australia; ${ }^{5}$ Deputy Nurse Co Director, Medical Division, Sir Charles

Gairdner Hospital, Perth, Western Australia 6009, Australia; ${ }^{6}$ Occupational Therapy Coordinator,

Acute Services Emergency Department, Sir

Charles Gardiner Hospital, Perth, Western

Australia 6009 , Australia; ${ }^{7}$ Registered Nurse, Sir

Charles Gairdner Hospital, Perth, Western

Australia 6009 , Australia; ${ }^{8}$ Project Officer, Nursing

Research, Perth Children's Hospital and Murdoch

University, Perth, Western Australia 6009,

Australia; ${ }^{\text {Project }}$ Officer, Centre for Nursing

Research, Sir Charles Gairdner Hospital, Perth,

Western Australia 6009 , Australia; ${ }^{10} \mathrm{National}$

Drug and Alcohol Research Centre, UNSW

Sydney, Sydney, New South Wales 2006, Australia;

"Rehabilitation, Ageing and Independent Living

(RAIL) Research Centre, Monash University,

Frankston, Victoria 3199, Australia

Correspondence: Christine Toye

School of Nursing, Midwifery and Paramedicine,

Curtin University, Perth, Western Australia

6102, Australia

Tel +6I 892661756

Email c.toye@curtin.edu.au
Background: Falls remain an important problem for older people in hospital, particularly those with high falls risk. This mixed methods study investigated the association between multiple bed moves and falls during hospitalisation of older patients identified as a fall risk, as well as safety of ward environments, and staff person-centredness and level of interprofessional collaboration.

Methods: Patients aged $\geq 70$ years, admitted through the Emergency Department (ED) and identified at high fall risk, who were admitted to four target medical wards, were followed until discharge or transfer to a non-study ward. Hospital administrative data (falls, length of stay [LoS], and bed moves) were collected. Ward environmental safety audits were conducted on the four wards, and staff completed person-centredness of care, and interprofessional collaboration surveys. Staff focus groups and patient interviews provided additional qualitative data about bed moves.

Results: From 486 ED tracked admissions, 397 patient records were included in comparisons between those who fell and those who did not [27 fallers/370 non-fallers (mean 84.8 years, SD 7.2; 57.4\% female)]. During hospitalisation, patients experienced one to eight bed moves (mean 2.0, SD 1.2). After adjusting for LoS, the number of bed moves after the move to the initial admitting ward was significantly associated with experiencing a fall (OR 1.56, 95\% CI 1.11-2.18). Ward environments had relatively few falls hazards identified, and staff surveys indicated components of person-centredness of care and interprofessional collaboration were rated as good overall, and comparable to other reported hospital data. Staff focus groups identified poor communication between discharging and admitting wards, and staff time pressures around bed moves as factors potentially increasing falls risk for involved patients. Patients reported bed moves increased their stress during an already challenging time.

Conclusion: Patients who are at high risk for falls admitted to hospital have an increased risk of falling associated with every additional bed move. Strategies are needed to minimise bed moves for patients who are at high risk for falls.

Keywords: inpatient, adverse events, older adults, falls, bed moves

\section{Introduction}

Older people are often at high risk of falls following admission to acute care hospitals. In Australia, data from 12 medical and surgical wards across six acute hospitals identified that $3.6 \%$ of the patients fell at least once, with almost one in three of these falls causing serious injuries. ${ }^{1}$ Patients who experienced an in-patient fall had an 8-day longer hospitalisation period than those who did not fall, and had 
an additional \$AUD6669 of hospital costs compared to non-fallers. ${ }^{1}$ Internationally, falls in United Kingdom hospitals over a 12-month period were estimated to cost $£ 15$ million, and resulted in 26 patient deaths, 500 hip fractures and 440 other fractures. ${ }^{2}$ This risk of falls is complex and can be due to a number of factors, and their interactions, including (1) the level of chronic falls risk an older person has (before their acute illness); (2) the effect of the acute illness resulting in hospital admission, which can magnify existing falls risk; (3) the new environment and routines associated with hospitals; and (4) staffing factors, including training and staff attitudes to ageing and falls. The extent of the problem of falls in hospitals has prompted the approach in the United States to reducing funding to hospitals for care provided for patients who fall while undergoing in-patient care. ${ }^{3}$ In Australia, a list of 16 Hospital-Acquired Complications (HACs) was developed by the Independent Hospital Pricing Authority (IHPA) and introduced in 2018, with in-patient falls resulting in fractures or intracranial injury rated second highest in impact on levels of reimbursement. ${ }^{4}$ Reimbursement for all HACs is incrementally reduced to reflect the additional costs incurred. In the case of falls, reimbursement is currently adjusted by approximately $2.5 \%$.

Effective falls prevention remains elusive in acute hospitals. A recent (2018) update of the Cochrane review evaluating interventions in hospital and residential care concluded that multifactorial intervention (addressing multiple falls risk factors, usually based on a falls risk assessment of individual patients) was the only intervention that may be effective in reducing falls rate. ${ }^{5}$ Only two of the five randomised trials included in this analysis of multifactorial interventions were effective in reducing fall rates, and both were in the sub-acute setting. Some relatively recent randomised trials that have shown effective interventions targeted (1) patient education in the acute or acute and sub-acute settings, particularly for cognitively intact patients $^{6}$ and (2) patient and staff education. ${ }^{7}$ However, other large recent trials using a multi-factorial approach were not effective in reducing falls or injuries. ${ }^{8}$ At the same time, some commonly applied approaches to reducing patient risk of falls have not been shown to be effective in large, well-designed randomised trials, for example, bed and chair alarms. ${ }^{9}$ Current research evidence provides limited guidance for hospital administrators, clinicians and researchers on how to successfully reduce risk of falls among older people in hospital.
The majority of falls prevention research in hospitals has focussed on single intervention approaches (environmental modification, exercise, sensor alarms) or combination (multifactorial) risk factor management. Care in acute hospitals is complex, and the focus of research to reduce falls has been on the individual patient within this complex system. ${ }^{5}$ However, there may be merit in also considering elements of the complex system interacting with patients, which may impact negatively (ie increase risk of falls) or positively (reduce risk of falls). For example, a relatively recent trend in hospitals hasincreased the number of bed moves for an individual patient during their hospitalisation. ${ }^{10,11}$ For the purposes of this paper, the definition of bed moves used by Ranasinghe et al was used - "all bed changes throughout a patient's admission, including changes between or within a given ward". ${ }^{12}$ Patients may be moved from their initial ward location for numerous reasons, including the need to accommodate another patient, transfer to "home ward", transfer to another specialist team, patient infection, transfer to intensive care, patient behaviours, need for closer observation, changing care needs of acutely ill, clinically deteriorating patient, and patient requiring palliative care. ${ }^{13,14}$ Increased numbers of bed moves have been reported to be associated with increased patient confusion; increased length of hospitalisation; and increased adverse clinical outcomes such as falls, medication error, or pressure ulcers. ${ }^{13}$ Patients with greater number of bed moves have been shown to have greater levels of cognitive impairment, delirium, and frailty. ${ }^{12,15}$ System or organisational factors other than bed moves that may also impact on individual patient care and safety include the person-centredness of care, ${ }^{16}$ level of inter-professional collaboration, ${ }^{17}$ and the safety of the ward environment. ${ }^{18-21}$

While there has been growing research in recent years evaluating bed moves, ward safety, and nature of care (including person-centredness), these have rarely been investigated in the one study, despite their potential interaction. This study was undertaken due to the strong association of falls with recurrent falls risk and the high personal and health system costs of falls in hospitals. ${ }^{1,22}$ In contrast to previous studies investigating bed moves in hospitals, the focus of this study was following the bed moves of admitted patients who were identified with a high risk for falls. The aims of this study were to evaluate in a high falls risk sample in an acute hospital (1) the frequency, impact and association with falls of multiple bed moves (move in the bed location of a patient); (2) the quality of ward environments, person-centredness, and level of inter-professional collaboration; (3) patients' perceptions of their within 
hospital transfers; and (4) staffs' perceptions of the implementation of falls prevention strategies in the ward, including the contributors and consequences of bed moves.

\section{Materials and Methods}

A mixed methods cohort study, including quantitative evaluation of the effect of bed moves on falls, and associated ward data, and a concurrent qualitative exploratory descriptive approach were conducted. The study was carried out in a tertiary teaching hospital with over 600 beds in Perth, Western Australia, between January and June 2016.

The study had three main components, including (1) tracking high falls risk patient transfers throughout the hospital (bed moves), from Emergency Department (ED) admission through four common transfer wards, until discharge; (2) evaluation of aspects of ward activity considered important in optimising patient care and safety, including (a) audits of ward environments; (b) evaluation of staff person-centredness and level of inter-professional collaboration; and (3) interviews with staff and patients in the four wards, investigating perspectives about bed moves.

The project was approved by the institutional Human Research Ethics Committees (Sir Charles Gairdner Group Human Research Ethics Committee - 2015-158; and Curtin University Human Research Ethics Committee HR219/2015). Written informed consent was provided for the staff focus groups and patient interviews. Collection of patient data from medical records was approved under a waiver of consent granted by the Human Research Ethics Committee. The application for waiver of consent was made on the basis of a) the very low risk inherent in the medical record audit; b) a belief that patients would be likely to provide consent if asked; and c) reducing participant burden by removing the need for inpatients to be approached at a time when they are likely to be feeling unwell and fatigued.

\section{Participants \\ Patients}

A total of 486 ED admissions included in this component of the study met the following inclusion criteria:

- aged 70 years and older;

- admitted via the ED to one of four general medical wards (Ward A [36 beds, providing care and assessment for up to $72 \mathrm{hrs}$ ]; Ward B [24 beds for general medical patients and 6 for patients with geriatric syndromes]; Ward C [20 beds for patients for evaluation of geriatric syndromes and 10 for older patients with acute delirium]; and Ward D [14-bed Geriatric Evaluation and Management Unit]);

- admitted because of a fall or assessed as being at high risk of falls by nursing staff and/or allied health staff in the ED or on admission, within $12 \mathrm{hrs}$ of arrival on the ward, using the items of the Falls Risk Assessment and Management Plan (FRAMP, part of a routinely used screening tool). ${ }^{23}$ Patients were rated as high risk of falls if they were scored as positive on at least three of the four routinely documented falls risk factors at the time of ED presentation or admission - (a) had a fall in the past 12 months; (b) unsteady when walking/transferring or uses a walking aid; (c) confused, known cognitive impairment, or incorrectly answers any of the following (age, date of birth, current year and place); and (d) has urinary or faecal frequency/urgency or nocturia.

Patients admitted from ED to a surgical or medical specialty ward were excluded.

Included patients remained in the study until they were discharged, moved into a non-study ward, or died.

\section{Staff}

Inclusion criteria for staff for the survey administration component of the study were that they provided direct patient care on the included wards as nurses, assistantsin nursing, student nurses, allied health professionals, allied health support staff, or doctors. Recruitment sought to include all eligible staff members in the participating wards in surveys via the distribution of named invitations.

Inclusion criteria for the nurse focus groups were all nursing staff and students working on the ward at the time at each group was held. Focus groups were scheduled during nursing shift overlap times to maximise opportunities for the staff to attend. Medical and allied health staff were excluded as the intended focus of the discussions was nursing practice.

\section{Procedures and Instruments Patient Tracking and Associated Data}

A patient log and tracking tool was completed by the project officer daily on weekdays, by reviewing the medical records to determine those meeting the study inclusion criteria. Weekend data were collected on Mondays. This patient $\log$ and tracking tool was used to document the basis for inclusion of each patient (ie, risk factors/admitted 
because of a fall or not); new falls risk assessments and related responses; bed moves (including the transfer from the ED to any participating ward, and then subsequent transfers to other participating wards, and moves within a ward), including dates of transfers to study and nonstudy wards, discharge, or death. Dates and times of any falls were also recorded along with (as available): the location of the fall, any resultant injuries, and the type of fall (eg, slip, trip). The study utilised the hospital definition of a fall, which was

an event which results in a person coming to rest inadvertently on the ground or floor or other lower level, other than as a consequence of sustaining a violent blow, loss of consciousness, sudden onset of paralysis as in stroke, or an epileptic seizure. $^{24}$

Bed moves were considered from ED transfer to the first ward and then within and between each included ward.

\section{Audits and Surveys}

Surveys and audits were conducted on all four wards at the start and the end of the study; however, only results from the initial evaluation are included in this paper.

\section{Ward Environmental Audit}

The Queensland Health general environmental audit for hospital wards was used to assess the physical environment of each of the four participating wards at the beginning of the study. This tool has been reviewed by the Victorian Quality Council and found to be applicable to the acute care setting, comprehensive, and suited to its purpose. ${ }^{25}$ The checklist addresses seven domains of the environment, five of which were relevant for this study: bathrooms and toilets (15 items), furniture (5 items), floor surfaces ( 8 items), lighting (14 items), and security of the environment (3 items). The checklist was completed by a member of the research team. Responses indicated compliance with generally accepted principles of falls risk minimisation (eg, lack of clutter) using a tick box format (yes, no, or not applicable). Positive (yes) scores (relating to desirable environmental characteristics) were scored as 0 , responses of "no" (relating to lack of desirable environmental characteristic/s) were scored as 1 . Scores for all items in each of the five environmental domains were summed. A score of 0 indicated no environmental hazards relating to that domain were identified, higher numbers indicated greater number of environmental risk factors identified.

\section{Staff Surveys}

Three questionnaires were distributed to all staff on participating wards prior to the commencement of the study (January 2016), to be returned via the internal mail or a box within the ward.

The person centeredness of staff on each ward was evaluated using two questionnaires: (1) the Person-Centred Health Care for Older Adults Survey (PCHCOAS), investigating 31 items with response options on a 5-point scale, Never (scored 1) to Always (scored 5). Scores were summed, with higher scores indicating higher level of staff person centredness care; and (2) the Person-centred care of Older People with cognitive impairment in Acute Care scale (POPAC). ${ }^{26}$ This 15 -item scale is focused more on care of cognitively impaired patients and has three sub-scales: (a) Using cognitive assessments and care interventions ( 5 items, 1-5); (b) Using evidence and expertise in cognition (3 items, 6-8); and (c) Individualizing care (7 items, 9-15). Response options range from Never (scored 1) to Always (scored 5), with higher scores indicating higher level of staff person centredness. Scores for each of the three sub-scales, and for the overall POPAC score were reported as an average (range 1-5).

An additional survey was used to evaluate the degree of the staff inter-professional collaboration, using the Assessment of Inter-Professional Team Collaboration Scale (AITCS). ${ }^{27}$ This scale has 37 items with response options on a 5-point scale [Never (scored 1) to Always (scored 5)]. Scores were averaged for the three sub-scales [Partnership (Items 1-19); Cooperation (Items 20-30); and Coordination (Items 31-37), and for total overall scale. Higher scores indicated higher level of inter-professional collaboration.

Interviews with Staff and Patients in the Four Wards A convenience sample of 21 staff from the participating wards agreed to participate in two focus groups. Ten nurses from Ward A participated in one focus group, and 11 nurses from Wards C and D, which were co-located, participated in a second focus group. Staff involved provided informed written consent. Issues addressed in the focus groups included how falls risk minimisation for older patients was implemented on the ward, what worked well and what could be improved, and how falls risk minimisation for this patient group impacted upon overall nursing clinical practice. Issues relating to patient bed moves were also explored. Focus groups were conducted by an experienced qualitative nurse researcher (SS) employed by the hospital in a non-clinical role. This researcher understood the hospital environment and was 
familiar to some participants but, having never practised on the wards, had no pre-existing working or management relationship with the attendees. Focus groups were conducted in a private room next to the ward clinical areas during the afternoon shift overlap time. Each lasted approximately 40 mins and was audio-recorded.

A purposeful sub-sample of patients (able to answer questions in English about their hospital stay and not experiencing uncorrected hearing loss) were invited to participate in interviews. Patients who were interviewed gave informed written consent. Patients were offered two options for the interview: (a) by telephone after discharge or (b) if requested by the patient, in a private area in the hospital immediately before discharge. Questions explored perceived risks of falling; communication with the staff related to falls risks; experiences related to falls risk management, in particular areas of the hospital and when being moved within the hospital.

\section{Data Management and Analysis}

Quantitative data were analysed using the $\mathrm{R}$ environment for statistical computing. ${ }^{28}$ For these analyses, only patients' first admissions during the study period were considered, and patients who were moved into wards not included in this study were removed from all summaries and analyses. Similarly, patients who were not discharged (or had not died) at the end of data collection, or for whom the date of the ED transfer could not be determined (eg, if this was immediately before or after midnight), were removed from all summaries and analyses.

Patient characteristics including age, gender, number of falls, length of stay, and the number of bed moves experienced were reported as mean (standard deviation [SD]) or median (range), depending on the nature and distribution of the data and normality of distribution, and the relationship between patient characteristics was assessed for multicollinearity. Logistic regression was used to determine factors associated with experiencing a fall during the hospital admission. Variables considered included age, gender, length of stay, number of moves during stay, whether the admission was falls-related, and whether the patient had a "companion" (employee remaining with the patient in an attempt to minimise risk of falls - also termed "sitters" during the admission. Variables significant at the 5\% level were retained in the final multivariate model. The only exceptions were length of stay and number of bed moves, which were forced into the model as these were the key variables of interest. Adjusted odds ratios (OR) and 95\% confidence intervals (CI) are provided. Model fitting measures, specifically Bayesian Information Criterion ${ }^{30}$ and Akaike's Information Criterion, ${ }^{31}$ are reported.

Generalised linear mixed effects models were used to investigate falls with respect to each bed move during the admission. Fixed effects included age, gender, number of days in the same location and ward as well as a random effect of patient ID. Variables significant at the 5\% level were retained in the final multivariate model and the only exceptions were length of stay and ward, which were forced into the model as these were the variables of interest. Adjusted OR (95\% CI) are provided. For the generalised linear mixed effects models, there were additional exclusions: patients who were discharged after the move from the ED to the first ward, and any patients for which the date of a bed move was unrecorded.

The average number of environmental hazard types identified in each ward for each of the five environmental domains (mean or median, depending on the nature of data distribution) and the most common hazards in each domain were reported. Mean and standard deviation measures for overall scales and sub-scales of the staff questionnaires were calculated for each ward, and averaged across the four participating wards.

All qualitative data were transcribed and subjected to thematic analysis. Transcriptions were checked for accuracy. Two members of the research team independently examined the transcripts line-by-line to identify significant words, phrases and sentences in the text and label as meaningful codes. Codes were grouped into tentative categories and described. Categories were grouped and organised as themes. Discrepancies between researchers' coding were resolved through discussion and returning to the data.

\section{Results}

\section{Evaluating the Frequency, Impact and Association of Multiple Bed Moves with Falls}

From 486 ED admissions tracked during the study who met inclusion criteria, there were 397 patients whose records were included in comparisons between those who fell and those who did not (27 fallers and 370 non-fallers, Figure 1 and Table 1). These patients were aged between 70 and 102 years (mean 84.8 years, SD 7.2), 57.4\% were female, and they had a median length of stay of 5.0 days (range 1-57 days). Thirty-eight percent of these 397 patients had a fall as their reason for admission. Patients 




Figure I Patients included in comparisons between fallers and non-fallers; and for the generalised linear mixed effects model analyses. Notes: *Two patients fell during these readmissions (and had not fallen on the first admission). **Two of these patients fell while in an included ward.

experienced between one and eight bed moves during their hospitalisation (mean 2.0, SD 1.2).

When investigating falls risk by ward, the ED was excluded as no falls occurred there. After excluding those patients (three of whom experienced a fall) who were discharged after the move from the ED to the first ward, and patients for which the date of a bed move was unrecorded, there were 220 patients with a total of 603 episodes of care in one bed space. During these episodes of care, 24 falls occurred. The percentage of episodes of care during which a fall was experienced was $2.2 \%$ for Ward A $(n=6), 4.6 \%$ for Ward $B(n=6), 6.4 \%(n=11)$ for Ward $\mathrm{C}$, and $4.6 \%$ in Ward D $(\mathrm{n}=1)$. After adjusting for length of stay, there was no significant difference in odds of falling between wards (all $\mathrm{p}>0.05$; results not shown). Fallers had a median length of stay (LoS) of
17 days (IQR 20) and a median of three bed moves during their hospitalisation (range 1-8) compared to the non-fallers [median LoS 5 days (IQR 8); median of two bed moves (range 1-7)]. After adjusting for length of stay, an increase in the number of bed moves during the hospital stay was associated an increased risk of falling (OR 1.56, 95\% CI 1.11-2.18) (Table 2).

\section{Comparing Ward Environments, Person-Centredness, and the Level of Inter-Professional Collaboration}

Environment

In each of the four wards, there were a small number of environmental hazards identified that may contribute to falls risk (Table 3). The floor surfaces and furniture domains each had a median of zero risk factors identified. 
Table I Clinical and Demographic Characteristics of the Patient Cohort

\begin{tabular}{|c|c|c|c|c|}
\hline & \multirow[t]{3}{*}{$\begin{array}{l}\text { Summary } \\
\text { Statistic }\end{array}$} & \multicolumn{2}{|c|}{$\begin{array}{l}\text { Experienced a Fall } \\
\text { During Hospitalisation }\end{array}$} & \multirow[t]{2}{*}{ Total } \\
\hline & & No & Yes & \\
\hline & & $(N=370)$ & $(\mathbf{N}=27)$ & $(N=397)$ \\
\hline Age & Mean $[S D]$ & $84.9[7.2]$ & $84.1[6.5]$ & $84.8[7.2]$ \\
\hline Female & $\mathrm{N}(\%)$ & $214(57.8 \%)$ & $14(51.9 \%)$ & $228(57.4 \%)$ \\
\hline Length of stay & Median [min-max] & $5[1-48]$ & $2[1-7]$ & $5[1-57]$ \\
\hline Number of bed moves & Median $[\min -\max ]$ & $17[4-57]$ & $3[1-8]$ & $2[1-8]$ \\
\hline \multicolumn{5}{|l|}{ Falls Risk Characteristics $\left(\mathrm{N}=396 ; 27\right.$ Individuals with a Fall) ${ }^{\mathrm{a}, \mathrm{b}}$} \\
\hline I. Had a fall in the past 12 months & $N(\%)$ & $326(88.4 \%)$ & $25(92.6 \%)$ & $351(88.6 \%)$ \\
\hline 2. Unsteady when walking/transferring or uses a walking aid & $N(\%)$ & $365(98.9 \%)$ & $27(100 \%)$ & $392(99.0 \%)$ \\
\hline 3. Confused, known cognitive impairment or incorrectly answers questions & $N(\%)$ & $267(72.4 \%)$ & $26(96.3 \%)$ & $293(74.0 \%)$ \\
\hline 4. Has urinary or faecal frequency/urgency of nocturia & $\mathrm{N}(\%)$ & $279(75.6 \%)$ & $20(74.1 \%)$ & $299(75.5 \%)$ \\
\hline $\begin{array}{l}\text { At least one of falls risk characteristics } 2,3 \text {, or } 4 \text { in any assessment during } \\
\text { hospitalisation }\end{array}$ & $\mathrm{N}(\%)$ & $368(99.7 \%)$ & $27(100 \%)$ & $395(99.8 \%)$ \\
\hline
\end{tabular}

Notes: andications of whether a fall was experienced in the past 12 months (falls risk characteristic I) represent records from risk assessments recorded either in the ED or first ward, or whether admission was related to a fall. For all other falls risk characteristics, the summaries represent the proportion of patients documented with the specific criteria on any assessment during hospitalisation. ${ }^{b}$ One patient was deemed suitable for inclusion in the study in the ED, although individual FRAMP criteria were not completed on the form.

Abbreviation: SD, standard deviation.

Table 2 Multivariate Logistic Regression Results: Experienced a Fall During Hospital Admission (Event=“Yes”)

\begin{tabular}{|l|l|l|l|}
\hline Variable & OR & $\mathbf{9 5 \% ~ C l ~}$ & P-Value \\
\hline $\begin{array}{l}\text { Length of Stay } \\
\text { One std dev (9.59 days) increase } \\
\text { Number of Moves During Stay } \\
\text { One move increase }\end{array}$ & 1.04 & $(0.99,1.09)$ & 0.076 \\
\hline & 1.56 & $(1.11,2.18)$ & 0.010 \\
\hline $\begin{array}{l}\text { Akaike's information criterion } \\
\text { Bayesian information criterion }\end{array}$ & & & Estimate \\
\hline
\end{tabular}

Abbreviations: $\mathrm{OR}$, odds ratio; $\mathrm{Cl}$, confidence interval.

There was some variability between wards for the environmental domains of bathroom and toilets, lighting, and security, although again there were relatively few environmental hazards identified for these domains (median number of environmental risk factors across the four wards of three (out of maximum 15), two (out of maximum 14) and two (out of maximum three) respectively. The most common environmental items identified in each domain are reported in Table 3.

\section{Staff Surveys}

Staff survey response rates ranged from 32\% (Ward A) to $47 \%$ (Ward D). The majority of the 105 responses were from registered nurses, enrolled nurses and clinical nurse specialists (69\%), although there were a small number of responses from medical (8.6\%) and allied health staff $(8.6 \%)$, and a small number of student nurses, allied health assistants, and others. The average age of respondents was 38.3 (SD 12.6) years, and respondents had worked an average of 48.4 months (44.2) on the ward they were currently working on.

Table 4 reports the summary scores for the three surveys (POPAC, PCHCOAS, and the AITCS) and subscales for the POPAC and AITCS, for each ward, and average scores across the four wards. Scores on the surveys were relatively consistent across the four wards. Overall, staff on the four wards demonstrated relatively high levels of person-centredness in their responses to the two questionnaires evaluating person-centredness, and comparable scores for the POPAC to another hospital staff sample in Sweden, ${ }^{32}$ and a lower overall score than another acute hospital study in Australia. ${ }^{33}$ Interprofessional collaboration was rated highly (mean score across the four wards across the three areas assessed of 150.1 (19.2), out of a maximum score of 185). Scores were comparable to a study from Sweden reporting AITCS scores, although the comparison sample was focussed on community teambased pain rehabilitation (no comparable hospital data for the AITCS were able to be identified). ${ }^{34}$ 
Table 3 Environmental Audit Scores (Median Number of Falls Risk Hazard Types Identified, and Most Common Hazards Identified)

\begin{tabular}{|c|c|c|c|c|c|c|}
\hline Environment & $\begin{array}{l}\text { Ward } \\
\text { A - Median }\end{array}$ & $\begin{array}{l}\text { Ward } \\
\text { B - Median }\end{array}$ & $\begin{array}{l}\text { Ward } \\
\text { C - Median }\end{array}$ & $\begin{array}{l}\text { Ward } \\
\text { D - Median }\end{array}$ & $\begin{array}{l}\text { Across All } 4 \\
\text { Wards - Median }\end{array}$ & $\begin{array}{l}\text { Items with Greatest Environmental Hazards } \\
\text { NOT Present - n (\%) }\end{array}$ \\
\hline $\begin{array}{l}\text { Bathrooms and Toilets; } \\
\text { (maximum } \\
\text { possible=15) }\end{array}$ & 3 & 3.5 & 1 & 2 & 3 & $\begin{array}{l}\text { - Availability of soap on a rope }(92.2 \%) \\
\text { - Shower chairs/commode with safety belt/safety } \\
\text { rail }(68.8 \%)\end{array}$ \\
\hline $\begin{array}{l}\text { Floor surfaces; } \\
\text { (maximum possible }=8 \text { ) }\end{array}$ & 0 & 0 & 0 & 0 & 0 & - Non-skid flooring (4.7\%) \\
\hline $\begin{array}{l}\text { Furniture; } \\
\text { (maximum possible=5) }\end{array}$ & 0 & 0 & 0 & 0 & 0 & \\
\hline $\begin{array}{l}\text { Lighting; } \\
\text { (maximum } \\
\text { possible=14) }\end{array}$ & 2 & 2 & 1 & 1 & 2 & $\begin{array}{l}\text { - Switches marked with luminous tape }(92.2 \%) \\
\text { - Firm \& colour contrasted handrails in passage- } \\
\text { ways }(45.3 \%)\end{array}$ \\
\hline $\begin{array}{l}\text { Security; } \\
\text { (maximum possible=3) }\end{array}$ & 2 & 3 & 1 & 2 & 2 & $\begin{array}{l}\text { - All exits secure to avoid confused patients leav- } \\
\text { ing (84.1\%) } \\
\text { - Clear walking routes where patients can walk } \\
\text { without becoming lost }(72.6 \%)\end{array}$ \\
\hline
\end{tabular}

\section{Staff and Patient Perceptions About Bed Moves and Falls Prevention Staff Focus Groups}

Two focus groups were held with 21 staff from three of the four participating wards. Fourteen participants were registered or enrolled nurses, four were clinical nurses, and three were student/assistant nurses (see Table 5 for details of participants). Half of the participants had more than 5 years clinical experience, and $87 \%$ had been working on the participating ward for more than 1 year. The focus groups covered a range of issues associated with managing patients with increased falls risk, including questions relating to patient bed moves and falls risk. Three themes emerged that described nurses' experiences of (1) resources to prevent falls, (2) communication about falls risks, and (3) factors influencing patient bed moves. Two of these themes pertain directly to the focus of this paper on patient bed moves (communication about falls risk and factors influencing patient bed moves) and are described below.

\section{Communication About Falls Risk}

(a) Communication, Risk Assessment and Care Planning. Communication between staff members, patients and family members was considered crucial to falls prevention practice. Nurses used information about the patient's characteristics and clinical condition to accurately assess the level of falls risk and determine appropriate prevention strategies. This communication was critical at times of staff movements, e.g. at shift changeover, and patient movements, e.g. between wards. In either scenario, a staff member was assuming care of an unfamiliar patient. Communication of pertinent information enabled the staff member to rapidly identify risks, and plan care to mitigate those risks. Whether communicated in writing or verbally, patient information such as “ ... a documented falls history, that's helpful rather than finding out yourself when they fall over" (Nurse \#1, FG1) was highly relevant. Additionally, nurses looked to the previous falls risk assessment communicated by the relinquishing staff member.

These nurses understood the risk of depending on inaccurate assessments. One nurse declared, "if we think a person is a 'one assist' because we've been told and really they are to 'two person [assist]' and we get them up and they fall" (Nurse \#1, FG2). The prime reason offered for potentially inaccurate reports communicated during these handovers was the lack of opportunity for comprehensive assessment in the previous care setting because nurses were, "not getting enough time to see the patient, know the patient very well" (Nurse \#2, FG1).

(b) Influence of Time Constraints. From the nurses' perspectives, the lack of time underpinning poor communication about falls risk was an inevitable consequence of a perceived pressure to move patients within and between wards. As evidence, one nurse recalled her experience of 
Table 4 Scores for the Person-Centred Care of Older People with Cognitive Impairment in Acute Care Scale (POPAC), the Person-Centred Health Care for Older Adults Survey (PCHCOAS), and the Assessment of Interprofessional Team Collaboration Scale.

\begin{tabular}{|c|c|c|c|c|c|c|c|}
\hline Assessment Tool & $\begin{array}{l}\text { Ward } \\
\text { A - Mean } \\
\text { (SD) }\end{array}$ & $\begin{array}{l}\text { Ward } \\
\text { B - Mean } \\
\text { (SD) }\end{array}$ & $\begin{array}{l}\text { Ward } \\
\text { C - Mean } \\
\text { (SD) }\end{array}$ & $\begin{array}{l}\text { Ward } \\
\text { D - Mean } \\
\text { (SD) }\end{array}$ & $\begin{array}{l}\text { Across All } 4 \\
\text { Wards - Mean } \\
\text { (SD) }\end{array}$ & \multicolumn{2}{|c|}{$\begin{array}{l}\text { Comparative } \\
\text { Data From } \\
\text { Other Studies }\end{array}$} \\
\hline \multicolumn{6}{|c|}{ Person-Centred Care of Older People with Cognitive Impairment in Acute Care Scale (POPAC) } & $\begin{array}{l}\text { Nilson } \\
\text { et } \text { al }^{32}- \\
\text { Sweden }\end{array}$ & $\begin{array}{l}\text { Grealish } \\
\text { et } \mathrm{al}^{33}- \\
\text { Australia }^{\mathrm{a}}\end{array}$ \\
\hline $\begin{array}{l}\text { Using cognitive assessments and care } \\
\text { interventions; } \\
(5 \text { items, rated I-5) - average score/ } \\
\text { item }\end{array}$ & $3.8(0.6)$ & $4.1(0.5)$ & $3.7(0.5)$ & $3.8(0.6)$ & $3.9(0.6)$ & $4.2(0.4)$ & $N / R$ \\
\hline $\begin{array}{l}\text { Using evidence and expertise in } \\
\text { cognition; } \\
\text { ( } 3 \text { items, rated I-5) - average score/ } \\
\text { item }\end{array}$ & $4.0(0.7)$ & $4.3(0.6)$ & $3.7(0.7)$ & $3.7(0.9)$ & $4.0(0.7)$ & $2.8(0.9)$ & $N / R$ \\
\hline $\begin{array}{l}\text { Individualizing care; } \\
\text { (7 items, rated I-5) - average score/ } \\
\text { item }\end{array}$ & $3.3(0.7)$ & $3.8(0.7)$ & $3.1(0.5)$ & $3.4(0.7)$ & $3.4(0.7)$ & $3.3(0.7)$ & $N / R$ \\
\hline $\begin{array}{l}\text { Total POPAC scores } \\
\text { (15 items, rated I-5) average score/item }\end{array}$ & $3.6(0.6)$ & $4.0(0.6)$ & $3.4(0.4)$ & $3.6(0.7)$ & $3.7(0.6)$ & $N / R$ & $4.4(0.58)$ \\
\hline \multicolumn{8}{|c|}{ Person-Centred Health Care for Older Adults Survey (PCHCOAS) } \\
\hline Total PCHCOAS score & $121.0(15.8)$ & $122.1(15.4)$ & $120.9(10.6)$ & $122.0(11.7)$ & $121.5(14.0)$ & $N / R$ & \\
\hline \multicolumn{6}{|c|}{ Assessment of Interprofessional Team Collaboration Scale } & \multicolumn{2}{|c|}{ Hellman et $a^{34}-S^{3}{ }^{2}$} \\
\hline $\begin{array}{l}\text { Partnership } \\
\text { (maximum possible score }=95 \text { ) }\end{array}$ & $78.6(7.6)$ & $78.5(12.7)$ & $75.3(8.1)$ & $76.5(10.6)$ & $77.6(9.9)$ & $74.5(15.2)$ & \\
\hline $\begin{array}{l}\text { Cooperation } \\
\text { (maximum possible score=55) }\end{array}$ & $46.7(5.4)$ & $45.4(6.8)$ & $41.9(3.4)$ & $45.0(6.7)$ & $45.3(6.1)$ & $44.7(7.2)$ & \\
\hline $\begin{array}{l}\text { Coordination } \\
\text { (maximum possible score }=35 \text { ) }\end{array}$ & $27.8(3.7)$ & $27.3(4.8)$ & $25.1(4.3)$ & $26.9(5.1)$ & $27.1(4.4)$ & $16.7(3.4)$ & \\
\hline $\begin{array}{l}\text { Total score } \\
\text { (maximum possible=|85) }\end{array}$ & $153.1(15.1)$ & $151.7(23.9)$ & $142.4(13.2)$ & $147.8(2 \mid .7)$ & 150.1 (19.2) & $N / R$ & \\
\hline
\end{tabular}

Notes: ${ }^{a}$ Comparison sample $=$ acute hospital medically oriented units. ${ }^{\mathrm{b}}$ Comparison sample $=$ community team-based pain rehabilitation. N/R - no comparison sample identified. Comparison data reported were available.

working on a medical ward which, by its nature, had a particularly high patient turnover:

When there is [sic] lots of patients in ED, they come in and within a few hours ... going to other ward ... I had the patient for two hours. I didn't even transfer the patient from bed. So, I have no idea about his mobility, even cognition ... so I was taking the patient to [another ward], I said "sorry guys, I cannot tell you how the patient ambulates. I have no idea". (Nurse \#2, FG2)

Another consequence of this perceived pressure was thought to be the potential for some staff members to limit the amount of information provided to receiving wards in order to secure acceptance of the patient. In response, one nurse called for more "honest" communication during handover such as

An approach to saying "look this person is a two person assist. They are heavy, they are unmotivated or ... they are a high falls risk" Don't sugar coat it. Just tell us the truth. We've got to work with it no matter what ... it helps us get prepared. (Nurse \#1, FG2)

This perceived pressure was thought to start in the ED and trickle down through the system. This nurse explained,

Each ward has pressures of their own to get that patient out ... it starts with the patient coming through Emergency [Department]. They need to get them in 
Table 5 Focus Group Participant Characteristics

\begin{tabular}{|c|c|}
\hline Participant Characteristic $(\mathrm{N}=\mathbf{2} \mathrm{I})$ & $\mathbf{N}(\%)$ \\
\hline \multicolumn{2}{|l|}{ Age (years) } \\
\hline $19-29$ & $5(23.8)$ \\
\hline $30-39$ & $3(14.3)$ \\
\hline $50-49$ & $5(23.8)$ \\
\hline $50-59$ & $3(14.3)$ \\
\hline $60+$ & $3(14.3)$ \\
\hline Missing & $2(9.5)$ \\
\hline \multicolumn{2}{|l|}{ Role } \\
\hline Staff Development Nurse or Clinical Nurse & $4(19)$ \\
\hline Registered Nurse or Enrolled Nurse & $14(66.7)$ \\
\hline Assistant in Nursing or Student Nurse & $3(14.3)$ \\
\hline \multicolumn{2}{|l|}{ Current Employment Status } \\
\hline Permanent full time & $9(42.9)$ \\
\hline Permanent part-time (8-9 days/fortnight) & $10(47.6)$ \\
\hline Permanent part-time ( $<8$ days/fortnight) & $0(0)$ \\
\hline Practicum & $2(9.5)$ \\
\hline \multicolumn{2}{|l|}{ Length of Time Employed on Current Ward (years) } \\
\hline$<1$ & $2(9.5)$ \\
\hline $1-3$ & $7(33.3)$ \\
\hline $4-6$ & $6(28.6)$ \\
\hline $7-9$ & I (4.8) \\
\hline $10+$ & $2(9.5)$ \\
\hline Missing or not applicable & $3(14.3)$ \\
\hline \multicolumn{2}{|l|}{ Length of Time in Current Profession (years) } \\
\hline$<1$ & $0(0)$ \\
\hline $1-5$ & $9(43)$ \\
\hline $6-10$ & $2(10)$ \\
\hline $11-15$ & $2(10)$ \\
\hline $16+$ & $5(24)$ \\
\hline Missing or not applicable & $3(13)$ \\
\hline \multicolumn{2}{|l|}{ Highest Level of Education } \\
\hline Diploma & 3 \\
\hline Bachelor degree & 13 \\
\hline Post-graduate qualification & 2 \\
\hline Missing or not applicable & 3 \\
\hline \multicolumn{2}{|l|}{ Ward (Nurses on All Participating Wards Invited) } \\
\hline A & 10 \\
\hline C or D & 11 \\
\hline
\end{tabular}

within their four-hour rule [author's note: a key performance indicator for Emergency Departments in Australia at the time of the study, relating to the proportion of patients discharged or admitted within four hours, termed colloquially the four-hour rule] up to a ward ... then they get put to another ward where there's a bed. They determine what their medical condition is so they go to the appropriate ward. They might get moved around if there is another person more severe that needs the bed so this person has to be relocated somewhere else (Nurse \#1, FG2) ... or there's a sick person who needs to go into a single room ... or there's an MRSA patient who needs to go into a single room. (Nurse \#3, FG2)

\section{Factors Influencing Patient Bed Moves}

When asked how decisions were made about moving patients within and between wards, nurses described patient and environmental characteristics that were considered influential. Several factors emerged as most influential. These included the need to manage competing clinical risks including falls risk, and sensitivity to patient preferences related to gender, age and cultural background. A nursing assessment that a patient was at risk of falling was considered to necessitate accommodation in a bed more visible to staff. However, rooms near the nursing station were a limited resource. The need to accommodate an acutely ill or infectious patient in a single or more visible room often meant relocating other patients within the ward. Poor communication in the clinical handover potentially increased the need to move patients when the receiving staff were provided insufficient information to plan appropriate accommodation. As this nurse explained,

We have extra moves generated by a lack of information from ... we would get someone come up [from ED] into a four-bedded room but they're droplet precautions. So then we've got to move someone out of a side [room]. That happens ... probably daily. (Nurse \#3, FG1)

Another potential outcome was that patients were inappropriately accommodated on arrival to the ward as nurses responded to pressure to take the admission. For the patient, this could mean that,

someone that might be a falls risk might get put into an inappropriate bed because we've got the pressure to get them up [from the ED to the ward], so they might go to somewhere far away from the nursing station.

(Nurse \#4, FG1)

The upshot was that the patient remained accommodated in an area where nurses had less oversight or experienced a move to a more suitable bed.

Lastly, accommodating patients of the same gender together, particularly to facilitate the comfort of older 
patients or for cultural reasons often directed bed moves.

As this nurse explained,

Most of the time, bed moves are done to ... have the same sex, gender [together] ... a lot of bed moves to put two female patients or two male patients together. To accommodate one patient, one female patient we would have had three bed moves ... some of the wards they do give, you know, all male and female patients together in rooms but here, [we have] elderly [patients] so we don't recommend that. (Nurse \#4, FG2)

\section{Patient Interviews}

Thirteen patients participated in the interviews ( 9 males, 11 were aged 80 years and over, and $54 \%$ were admitted due to a fall). The interview data indicated that it was difficult for patients to separate their experiences of falls prevention from the overall experience of illness, hospital admission and falls experienced prior to presenting at ED. Several concepts were identified in the data, but the descriptions were thin and saturation was not reached.

One patient reported not feeling at risk of falling but then having a fall in hospital, which she attributed to the actions of others. She recalled, "slid[ing] out of the commode in the shower because I was unattended ..." (P4). Most participants, however, reported feeling safe while in hospital because they took precautions, by accessing safety equipment and staff support.

The other emergent theme related to patients' perceptions of their moves during the hospital admission. Several patients indicated that moving between wards and beds increased the already stressful nature of a hospital admission.

One patient remarked, “... hospitals are distressing enough without having to be moved around" (P4). There was a suggestion that poor communication may have added to the stress of moving beds for these patients, as evidenced by one patient who asked, "why the hell do they keep moving you from room to room ... it is the first room, they say you've contaminated it" (P8), while others recalled, "If I've asked they've just told me that I'm being moved to another ward but they haven't given any reason why .... I've just been exhausted when I've been moved and that's it" (P3).

\section{Discussion}

The results of this study highlight that older people with high falls risk being admitted to an acute hospital have an average of two bed transfers during their admission, and that every bed move is associated with $56 \%$ increase in odds of falling. Patient interviews and staff focus groups reinforced the added burden they each perceived associated with bed transfers, and staff focus groups identified factors they considered contributed to increased falls risk with bed transfers, including time constraints, and lack of detail or inaccurate details provided in handover between the bed transfer wards. The implications of these findings are that questions need to be asked relating to the frequency and rationale for all of the bed moves occurring (in this study, one high falls risk older patient experienced eight bed moves during her single hospitalisation episode). It is clear that some bed moves are necessary for patient flow, infection control, specialist management and other reasons. However, strategies need to be considered to determine if bed moves might be able to be reduced, particularly for patients who are at high risk for falls. The study also evaluated factors considered to possibly impact on falls risk and bed transfers, including safety of ward environments (generally the participating wards had low levels of environmental falls risk), and the level of person-centredness and collaborative care of staff on participating wards (overall ratings indicated generally high level of person-centred care and collaborative approach to care by staff).

A small number of other studies have investigated the effects of bed moves for older hospitalised patients and identified similar trends in terms of falls and other adverse outcomes for patients. Most of these previous studies have not focussed specifically on patients who are at high risk for falls (as our study did). Ranasinghe et al identified worse outcomes (eg increased dependence, discharge to residential care, or death) for patients under an Older Person Evaluation Review and Assessment team management (who had a significantly higher frequency of bed moves, although higher levels of comorbidities), relative to General Medicine patients. ${ }^{12}$ Another study of patients admitted to a tertiary referral hospital (mean age 58.1 years) identified that $40.6 \%$ of the patients had at least one bed move during their hospitalisation, and that three or more bed moves (experienced by $4.9 \%$ of the sample) significantly increased the risk of an adverse event (including falls, but also other adverse outcomes such as medication error or pressure ulcer) nearly threefold. ${ }^{13}$ These studies have most likely under-estimated the impact of bed moves because their sampling has not focussed on patients who are at high risk for falls, and the latter study did not include cognitively impaired patients. Our 
study adds to these existing studies in that the sample was aged $\geq 70$ years (mean age 85 ), only included patients who are at high risk for falls, and also included both cognitively intact and cognitively impaired patients, through a waiver of consent approved through the Ethics Committee.

The staff interviews identified inadequate transfer communication from the discharging ward to the receiving ward, and time pressures for nursing staff in preparing for bed moves as factors contributing to increased risk of falls for patients after such a move. Studies have highlighted some of the factors associated with triggering bed moves, with common factors for within ward bed moves including [in reducing order] bed needed for another patient, patient request, patient infection, and need for closer observation; and common factors triggering between ward transfers being transfer to home ward, transfer from short stay unit, and transfer to specialist unit. ${ }^{13}$ In another study investigating decision-making regarding moving patients into single rooms (one purpose of bed moves), Bloomer et al identified that bed moves commonly occur as a consequence of changing need, and contributory factors included infection control, and patient behaviour (such as agitation and aggression). ${ }^{14}$ This study also highlighted staff perceptions of the negative aspects of bed moves, which included increased patient disorientation, and workload of support staff (including cleaning, equipment relocation, etc.). ${ }^{14}$ In the present study, within ward bed moves were also highlighted as an issue, often involving moving patients who are at high risk for falls with cognitive impairment to "more visible" areas of the ward (often close to the nurses' station).

In this study, we have attempted to draw together a focus on a number of different domains that can impact on quality of care, so that these factors can be viewed together with our primary focus on bed moves and falls risk, as linked elements to optimise patient outcomes during hospitalisation. Person-centred care is increasingly being recognised as an essential element of quality care and improved patient outcomes for older hospital inpatients, even more so for older patients with cognitive impairment. $^{32,33,35}$ The PCHCOAS tool (for use with older patients generally) ${ }^{35}$ and the POPAC tool (particularly targeting person-centred care for older patients with cognitive impairment $)^{32,33}$ can be used to quantify the person-centredness of staff at a given point in time, or longitudinally, particularly monitoring change over time, for example, after training or other initiatives to improve person-centred care, or high levels of staff turnover.
Additional factors such as environmental safety and greater levels of interprofessional collaboration are also key factors in quality care. Data in the present study indicated that although overall performance on these measures was relatively good, there remained scope for improvement. However, there are only limited comparison data available in acute hospitals identified for the POPAC (another Australian study, and a study from Sweden), and the Assessment of Interprofessional Team Collaboration Scale (comparison study from Sweden), which may limit the interpretation of this comparative data, due to international differences in hospital systems and models of care. Lower performance on these domains may increase falls risk and contribute to the overall system or organisation factors (including bed moves) to be considered in improving care and reducing falls among older hospitalised patients. Intermittent assessment of these domains, and actions to address areas identified of concern, should be part of routine care.

The built environment is a core aspect of patient safety, falls risk, ${ }^{8,20}$ and potentially also may influence positively or negatively the need for bed moves. Changes to ward layout or practice that can accommodate increased visibility for patients with high falls risk, for example, through decentralized nursing or portable nursing stations, ${ }^{36}$ may reduce risk of falls, and the need for bed moves. The use of acuity adaptable rooms that provide a greater flexibility for managing complex and changing patient needs within a single location has been shown in a pre-post design study to reduce bed moves, medication errors and falls, as well as other organisational, staff and patient benefits. ${ }^{37}$ Although one component of our study focussed on some of the environmental and ward layout factors that may contribute to falls risk, there may be broader considerations not included in our environmental survey that should be considered. Hignett ${ }^{38}$ highlights that although building design, layout, décor, signage and lighting levels are examples of essential components of this, that hospital room and ward design also needs to be centred around the key organisational, technology, and user (staff - across all clinical and non-clinical areas; and patients and families) interface with the environment are also critical to a successful model. A recent integrative review highlighted the importance of the built environment in hospitals as a contributor to falls risk, but that there is limited research quantity and quality to adequately inform healthcare design decision-making. ${ }^{20}$ Further research needs to focus on design elements for hospital room and wards that 
can be implemented to achieve improved organisational, staff and patient outcomes, ${ }^{8}$ including reduced falls and reduced bed moves.

Given the complex and often rapidly changing status of patients and patient mix on any given ward, and that some bed moves will always be required, a key question to consider is whether some bed moves could be avoided, or whether the individual/s being moved could be those with lower level of falls risk, and without delirium or dementia. For example, one option would be to aim to prioritise consistent use of rooms closest to nurses stations for patients with high falls risk and/or delirium or dementia, and try to minimise transfers of a high falls risk or cognitively impaired patient to a more distant room in the first instance, and requiring a second move once the magnitude of the issue of falls risk or cognitive impairment is evident on the receiving ward. Another strategy may be to reduce use of short-term acute management of the elderly type wards (with expectations of short length of stay and discharge) for patients who are not clearly likely to be discharged in the required short time frame, and thereby discharge from the ED directly to a standard ward (avoiding one bed move from the interim ward). Using a prospective study with historical controls, a structured approach to fast track targeted patients directly from the ED to a Geriatric Evaluation and Management ward (thereby reducing use of interim ward moves), combined with related strategies including standardised assessment of cognition, medications, and mobility and discharge risk, and access to a falls registrar and geriatric consultant has been shown to reduce bed moves and complication rates. ${ }^{39}$ Some similar strategies have been introduced in the participating hospital subsequent to our study, due to the increased awareness of the associations between bed moves, falls risk, and delirium or cognitive impairment. Further research of innovative approaches and evaluation of protocols to minimise bed moves for patients who are at high risk for falls are clearly warranted.

Clinical implications from the study outcomes include (1) that bed moves for patients who are at high risk for falls should be minimised, that is, the risks from them should be balanced against likely benefits. Where there are options, consideration of moving a patient with lower falls risk should be considered. Staff education may be needed to ensure that there is an awareness of this issue; (2) when bed moves are deemed necessary for patients who are at high risk for falls, it is imperative that staff recognise the increased risk of falls after a bed move, and take action to mitigate this; (3) communication among the staff (from discharging ward to receiving ward) about the patient's falls risk should be clear and comprehensive. One way in which this might be supported would be to use the Falls Risk Assessment and Management Plan (or similar falls risk tool) as a prompt; and (4) there should be an awareness - across all staff members delivering patient care - that falls risk minimisation is a joint responsibility among all staff and professional groups, and associated education may be required to ensure this.

A strength of this study was using mixed methods, including a qualitative investigation of the perceptions of both staff and patients on factors influencing bed moves. Although not reaching saturation, the patient interviews highlighted the high stress for older patients about the hospitalisation experience, and that they considered bed moves to add to this stress. This added stress may also be a contributory factor in increased falls risk after a bed move. Timing of bed moves also seemed to be a further stress, particularly if occurring overnight. If a bed move is necessary, then staff should take time to explain the reason for the move, and if possible, do this during daytime hours. Although these results should be considered preliminary because of not reaching saturation, patient perspectives are an essential consideration, and warrant further research exploration with a larger sample.

This study had several limitations. The study was conducted in one division of a single hospital, meaning that findings may not be generalizable. Data were censored when individuals were transferred out of the four participating wards, which may have resulted in a lower number of falls reported than actually occurred during these patient's complete episodes of care. Survey responses were relatively low, and although a range of staff including medical, nursing and allied health staff on the study wards were targeted, responses were primarily from nursing staff. Finally, although the study aimed to reach saturation with the two qualitative components, this was not achieved from the patient sample ( $\mathrm{n}=13)$, so these results need to be interpreted with caution. The impact of patient acuity on falls risk and bed moves could not be accounted for in this study because of the limited measures of patient acuity assessed, and that all included patients had at least three of the four falls risk characteristics present (used in this study as indicators of acuity of falls risk). Future studies should explore in greater detail the influence of patient acuity and falls risk and bed moves. 


\section{Conclusions}

Each patient bed move increased the odds of a fall in hospital in high falls risk hospitalised patients by $56 \%$. Inadequate communication between transferring and receiving wards, and staff time pressures at the time of bed moves were considered as main contributors to this increased risk of falls after a patient bed move. Acute hospitals should explore strategies to minimise bed moves, particularly for patients who are at high risk for falls, and if bed moves do need to occur, to explore options for moving lower falls risk patients to address the bed move need where possible.

\section{Funding}

Funding was received from Sir Charles Gairdner Hospital and the School of Nursing, Midwifery and Paramedicine, Curtin University.

\section{Disclosure}

The authors report no conflicts of interest in this work.

\section{References}

1. Morello RT, Barker AL, Watts JJ, et al. The extra resource burden of in-hospital falls: a cost of falls study. Med J Aust. 2015;203(9):367. doi:10.5694/mja2.2015.203.issue-9

2. NHS national patient safety agency: the third report from the patient safety observatory: slips, trips and falls in hospital (PSO3 Summary). 2007.

3. Inouye SK, Brown CJ, Tinetti ME. Medicare nonpayment, hospital falls, and unintended consequences. N Engl J Med. 2009;360 (23):2390-2393. doi:10.1056/NEJMp0900963

4. Hospital-acquired complications (HACs). Available from: https:// www.safetyandquality.gov.au/our-work/indicators/hospital-acquiredcomplications. Accessed November 25, 2019.

5. Cameron ID, Dyer SM, Panagoda CE, et al. Interventions for preventing falls in older people in care facilities and hospitals. Cochrane Database Syst Rev. 2018;9:Cd005465.

6. Haines TP, Hill AM, Hill KD, et al. Patient education to prevent falls among older hospital inpatients: a randomized controlled trial. Arch Intern Med. 2011;171(6):516-524. doi:10.1001/archinternmed.2010.444

7. Hill AM, McPhail SM, Waldron N, et al. Fall rates in hospital rehabilitation units after individualised patient and staff education programmes: a pragmatic, stepped-wedge, cluster-randomised controlled trial. Lancet. 2015;385(9987):2592-2599. doi:10.1016/S01406736(14)61945-0

8. Barker AL, Morello RT, Wolfe R, et al. 6-PACK programme to decrease fall injuries in acute hospitals: cluster randomised controlled trial. BMJ. 2016;352:h6781. doi:10.1136/bmj.h6781

9. Shorr RI, Chandler AM, Mion LC, et al. Effects of an intervention to increase bed alarm use to prevent falls in hospitalized patients: a cluster randomized trial. Ann Intern Med. 2012;157(10):692-699. doi:10.7326/0003-4819-157-10-201211200-00005

10. Blay N, Duffield CM, Gallagher R. Patient transfers in Australia: implications for nursing workload and patient outcomes. $J$ Nurs Manag. 2012;20(3):302-310. doi:10.1111/jonm.2012.20.issue-3

11. Blay N, Roche MA, Duffield C, Gallagher R. Intrahospital transfers and the impact on nursing workload. J Clin Nurs. 2017;26(23-24):4822-4829. doi:10.1111/jocn. 13838
12. Ranasinghe C, Fleury A, Peel NM, Hubbard RE. Frailty and adverse outcomes: impact of multiple bed moves for older inpatients. Int Psychogeriatr. 2017;29(2):345-349. doi:10.1017/S104161021600 1605

13. Webster J, New K, Fenn M, et al. Effects of frequent PATient moves on patient outcomes in a large tertiary Hospital (the PATH study): a prospective cohort study. Aust Health Rev. 2016;40(3):324-329. doi:10.1071/AH15095

14. Bloomer MJ, Lee SF, Lewis DP, Biro MA, Moss C. Single-room usage patterns and allocation decision-making in an Australian public hospital: a sequential exploratory study. J Clin Nurs. 2016;25(15-16):2200-2210. doi:10.1111/jocn.13264

15. Goldberg A, Straus SE, Hamid JS, Wong CL. Room transfers and the risk of delirium incidence amongst hospitalized elderly medical patients: a case-control study. BMC Geriatr. 2015;15:69. doi:10.11 86/s12877-015-0070-8

16. Alsalem G, Bowie P, Morrison J. Assessing safety climate in acute hospital settings: a systematic review of the adequacy of the psychometric properties of survey measurement tools. BMC Health Serv Res. 2018;18(1):353. doi:10.1186/s12913-018-3167-x

17. Walters SJ, Stern C, Robertson-Malt S. The measurement of collaboration within healthcare settings: a systematic review of measurement properties of instruments. JBI Database System Rev Implement Rep. 2016;14(4):138-197. doi:10.11124/JBISRIR-2016-2159

18. Tzeng HM, Yin CY. The extrinsic risk factors for inpatient falls in hospital patient rooms. J Nurs Care Qual. 2008;23(3):233-241. doi:10.1097/01.NCQ.0000324588.91837.84

19. Choi YS, Lawler E, Boenecke CA, Ponatoski ER, Zimring CM. Developing a multi-systemic fall prevention model, incorporating the physical environment, the care process and technology: a systematic review. $J$ Adv Nurs. 2011;67(12):2501-2524. doi:10.1111/j.13652648.2011.05672.x

20. Pati D, Valipoor S, Lorusso L, et al. The Impact of the built environment on patient falls in hospital rooms: an integrative review. J Patient Saf. 2019. doi:10.1097/PTS.00000000000006613

21. Taylor E, Hignett S. The SCOPE of hospital falls: a systematic mixed studies review. Herd. 2016;9(4):86-109. doi:10.1177/1937586716645918

22. Deandrea S, Lucenteforte E, Bravi F, Foschi R, La Vecchia C, Negri E. Risk factors for falls in community-dwelling older people: a systematic review and meta-analysis. Epidemiology. 2010;21 (5):658-668. doi:10.1097/EDE.0b013e3181e89905

23. Western Australian Department of Health. Development of Falls Risk Assessment and Management Plan. Perth: Health Strategy and Networks Branch, Western Australian Department of Health; 2015. Available from: https://ww2.health.wa.gov.au/ /media/Files/ Corporate/general\%20documents/falls/PDF/Dev-of-FRAMP.pdf. Accessed April 12, 2019.

24. Gibson M, Andres R, Isaacs B, Radebaugh T, Worm-Peterson J. The prevention of falls in later life. A report of the kellogg international working group on the prevention of falls by the elderly. Dan Med Bull. 1987;34(Supp 4):1-24.

25. Victorian Quality Council - Government of Victoria: Minimising the risk of falls and fall-related injuries. Melbourne, Australia: Government of Victoria; 2004.

26. Edvardsson D, Nilsson A, Fetherstonhaugh D, Nay R, Crowe S. The person-centred care of older people with cognitive impairment in acute care scale (POPAC). J Nurs Manag. 2013;21(1):79-86. doi:10.1111/jonm.2013.21.issue-1

27. Orchard C, King G, Khalili H, Bezzina M. Assessment of interprofessional team collaboration scale (AITCS): development and testing. J Contin Educ Health Prof. 2012;32(1):58-67. doi:10.1002/chp.21123

28. R Core Team: R. A Language and Environment for Statistical Computing. Vienna, Austria: $\mathrm{R}$ Foundation for Statistical Computing; 2018. Available from:: https://www.R-project.org/. Accessed April 12, 2019. 
29. de Jong LD, Kitchen S, Foo Z, Hill AM. Exploring falls prevention capabilities, barriers and training needs among patient sitters in a hospital setting: a pilot survey. Geriatr Nurs. 2018;39(3):263-270. doi:10.1016/j.gerinurse.2017.09.006

30. Schwarz G. Estimating the dimension of a model. Ann Stat. 1978;6:461-464. doi:10.1214/aos/1176344136

31. Akaike H. A new look at the statistical model identification. IEEE Trans Automat Contr. 1974;19:716-723. doi:10.1109/TAC.1974.110 0705

32. Nilsson A, Lindkvist $M$, Rasmussen BH, Edvardsson D. Measuring levels of person-centeredness in acute care of older people with cognitive impairment: evaluation of the POPAC scale. BMC Health Serv Res. 2013;13:327. doi:10.1186/14726963-13-327

33. Grealish L, Chaboyer W, Harbeck E, Edvardsson D. The personcentred care of older people with cognitive impairment in acute care (POPAC) scale - psychometric evaluation. J Nurs Manag. 2017;25 (2):139-147. doi:10.1111/jonm.2017.25.issue-2

34. Hellman T, Jensen I, Orchard C, Bergstrom G. Preliminary testing of the Swedish version of the assessment of interprofessional team collaboration scale (AITCS-S). $J$ Interprof Care. 2016;30 (4):499-504. doi:10.3109/13561820.2016.1159184
35. Dow B, Fearn M, Haralambous B, Tinney J, Hill K, Gibson S. Development and initial testing of the person-centred health care for older adults survey. Int Psychogeriatr. 2013;25(7):1065-1076. doi:10.1017/S1041610213000471

36. Ali UM, Judge A, Foster C, et al. Do portable nursing stations within bays of hospital wards reduce the rate of inpatient falls? An interrupted time-series analysis. Age Ageing. 2018;47(6):818-824 doi:10.1093/ageing/afy097

37. Hendrich AL, Fay J, Sorrells AK. Effects of acuity-adaptable rooms on flow of patients and delivery of care. Am J Crit Care. 2004;13 (1):35-45.

38. Hignett S. Why Design Starts with People. The Health Foundation: Inspiring Improvement; 2013:1-5.

39. Wahbi O, Mandal B, Dumas S, Leyte N, Arendts G. Configuration of a standardized approach to patients $>65$ years old presenting to emergency department with a fall. in: Abstracts of the 34th annual scientific meeting of the Australasian College for Emergency Medicine. Emerg Care Australas. 2018;30(S1):19-20.
Clinical Interventions in Aging

\section{Publish your work in this journal}

Clinical Interventions in Aging is an international, peer-reviewed journal focusing on evidence-based reports on the value or lack thereof of treatments intended to prevent or delay the onset of maladaptive correlates of aging in human beings. This journal is indexed on PubMed Central, MedLine, CAS, Scopus and the Elsevier

\section{Dovepress}

Bibliographic databases. The manuscript management system is completely online and includes a very quick and fair peer-review system, which is all easy to use. Visit http://www.dovepress.com/ testimonials.php to read real quotes from published authors. 